\title{
REDUCCIÓN DE LOS TIEMPOS DE ADQUISICIÓN DE IMÁGENES POR RESONANCIA MAGNÉTICA UTILIZANDO TÉCNICAS DE COMPRESSED SENSING
}

Carlos Sing-Long C(1), Cristián Tejos N, Ph.D.(2), Pablo Irarrazaval M, Ph. $D^{(3)}$.

1. Ingeniero Civil Electricista, Investigador, Departamento de Ingeniería Eléctrica, Pontificia Universidad Católica de Chile, Centro de Imágenes Biomédicas, Pontificia Universidad Católica de Chile.

2. Profesor Asistente Adjunto, Departamento de Ingeniería Eléctrica, Pontificia Universidad Católica de Chile, Centro de Imágenes Biomédicas, Pontificia Universidad Católica de Chile.

3. Profesor Titular, Departamento de Ingeniería Eléctrica, Pontificia Universidad Católica de Chile, Centro de Imágenes Biomédicas, Pontificia Universidad Católica de Chile.

Este trabajo fue efectuado en el Departamento de Ingeniería Eléctrica, Pontificia Universidad Católica de Chile y el Centro de Imágenes Biomédicas, Pontificia Universidad Católica de Chile, Av. Vicuña Mackenna 4860, Macul, Santiago, ctejos@ing.puc.cl.

\section{REDUCTION OF THE ACQUISITION TIME IN MAGNETIC RESONANCE IMAGING USING COMPRESSED SENSING}

\begin{abstract}
Introduction: The acquisition process in Magnetic Resonance Images (MRI) is slow. One approach to reduce the acquisition times is the reconstruction of undersampled data. i.e. to acquire less samples that those needed for standard application, and to reconstruct the unknown samples using mathematical algorithms. We propose to used reconstruction techniques for undersampled data based on Compressed Sensing (CS) to decrease the acquisition times, obtaining identical MRI as those obtained with all samples. Methods: We performed reconstructions of undersampled data obtained from phantoms and MRI with $60 \%, 55 \%$ and $50 \%$ of the samples. Results: When the number of samples was more that the double of pixels with non cero intensity, the reconstructions where identical to the original ones. For the MRI experiment, this was achieved with $60 \%$ of the samples, therefore obtaining a $40 \%$ of reduction in the acquisition time. Discussion: Our reconstruction technique based on CS is an effective way for reducing the acquisition times in MRI.
\end{abstract}

\section{RESUMEN}

Introducción: El proceso de adquisición de imágenes por resonancia magnética (IRM) es lento. Una forma para disminuir los tiempos de adquisición es a través de reconstrucciones de datos submuestreados, es decir tomar menos muestras que las necesarias en aplicaciones estándares, y reconstruir las muestras faltantes a través de algoritmos matemáticos. Proponemos utilizar técnicas de reconstrucción de datos submuestreados basadas en técnicas de Compressed Sensing (CS) para disminuir los tiempos de adquisición, obteniendo imágenes idénticas a las obtenidas con todas las muestras. Métodos: Realizamos reconstrucciones de datos submuestreados de fantomas y IRM con $60 \%, 55 \%$ y $50 \%$ de las muestras. Resultados: Cuando el número de muestras fue mayor al doble del número de pixeles con intensidad cero, las reconstrucciones obtenidas fueron idénticas a las originales. Para las IRM esto se logró con $60 \%$ de las muestras, logrando reducciones del $40 \%$ en los tiempos de adquisición. Discusión: Nuestra técnica de reconstrucción basada en CS es una forma efectiva para reducir los tiempos de adquisición de IRM.

Palabras clave: Magnetic resonance imaging, Image reconstruction, Compressed sensing. 


\section{INTRODUCCIÓN}

La formación de imágenes por resonancia magnética (MRI, del inglés Magnetic Resonance Imaging) es una técnica no invasiva utilizada ampliamente para obtener imágenes de alto contraste en una gran variedad de tejidos. Sin embargo, el tiempo necesario para adquirir una imagen es largo y se incrementa en forma proporcional al aumento de ciertos parámetros que definen la calidad deseada (e.g. la resolución, el número de promedios para mejorar la razón señal a ruido), de modo que existe un compromiso entre calidad y duración del examen. Diversos métodos han sido propuestos para reducir el tiempo de adquisición de una imagen, entre los más populares están aquellos adquieren menos muestras que las necesarias para reconstruir una imagen estándar, y luego aplican algún algoritmo matemático para estimar los datos no adquiridos. Estos métodos buscan reconstruir una imagen que satisfaga un cierto criterio o propiedad y que conserve los valores de las muestras que fueron adquiridas, condición que se denomina consistencia de datos. En estos métodos hay generalmente un compromiso entre la exactitud de la imagen obtenida (i.e. qué tan parecida es a la imagen que se hubiera obtenido con todas las muestras) y la tasa de submuestreo utilizada (i.e. la proporción entre el número de muestras que idealmente debiéramos haber obtenido y número de muestras adquiridas). A las imágenes obtenidas a través de este tipo de técnicas se les denomina imágenes submuestradas.

Compressed Sensing (CS) ${ }^{(1)}$ es una técnica creada para reconstruir señales en general (audio, imágenes, etc.) que han sido submuestreadas y fue utilizada por primera vez como una técnica de reconstrucción de imágenes submuestreadas de MRI por Lustig et al. ${ }^{(2)}$. Esta técnica funciona para reconstruir imágenes que puedan ser representadas con pocos coeficientes, es decir que hay muchos lugares donde la imagen es igual a cero. Esto sucede frecuentemente en las imágenes de MRI, por ejemplo alrededor del paciente, ya que el aire no produce señal alguna y vemos por lo general un fondo negro. El método de reconstrucción de CS de una imagen submuestreada consiste en probar distintas imágenes que cumplan con la consistencia de datos, y elegir aquella imagen te tenga la mayor cantidad de pixeles iguales a cero, proceso al que se le conoce como minimización de la cuasi-norma $\mathrm{I}_{0}$ de una imagen. Está demostrado matemáticamente que si se adquieren más muestras que el doble del número de pixeles diferentes de cero de la imagen (independiente de cuales sean las muestras adquiridas), entonces la imagen reconstruida será exacta, i.e. idéntica a la que se obtendría de obtener todas las muestras. Para dar un ejemplo, esto significa que si tengo una imagen con 100 pixeles, de los que se sabe que hay sólo 40 distintos de cero (pero no se sabe dónde están ubicados), me bastaría adquirir 80 muestras para obtener la imagen ideal, y por consiguiente puedo reducir el tiempo de adquisición en un $20 \%$.

Desafortunadamente, no existe una metodología que permita encontrar de forma óptima la imagen que satisfaga esta minimización y la única manera de encontrar la solución es probando todas las posibles combinaciones (formalmente se llama a esto un problema NP-completo). Luego, el tiempo requerido para reconstruir la imágen a partir de las muestras adquiridas es prohibitivo. Por esta razón, Candes et al. ${ }^{(1)}$ modificaron el proceso de minimización de la cuasi-norma $\mathrm{I}_{0}$ en CS por una minimización de la norma $I_{1}$ de las imágenes, i.e. minimizar la suma de las intensidades de todos los pixeles de la imagen, sujeto a la consistencia de datos. Este nuevo método de CS puede ser resuelto con programas computacionales estándares, sin embargo, es necesario ahora conocer una cantidad de muestras considerablemente mayor que en el caso anterior (las tasas de submuestreo que pueden lograrse son menores) y en algunos casos, aún cuando se cumpla el requerimiento del número de muestras adquiridas, puede que la imagen reconstruida no sea idéntica a la ideal.

Para aprovechar sus ventajas, Trzasko et al. ${ }^{(3)}$ retomaron el enfoque minimización de la cuasi-norma $\mathrm{I}_{0}$ en CS y lograron expresarlo como un problema de minimización convencional y factible de solucionar utilizando aproximaciones con funciones continuas. Sus resultados experimentales con MRI muestran que ésta es una técnica de reconstrucción efectiva y permite tasas de submuestreo mayores a las conseguidas en CS con la norma $\mathrm{I}_{1}$. Adicionalmente, Mohimani et al. ${ }^{(4)}$ demostraron que la reconstrucción a través de este método es siempre exacta si se adquieren suficientes muestras. Sin embargo, el método de Trzasko et al. aún posee ciertas dificultades, ya que depende de algunos parámetros cuyos valores no son fáciles de encontrar.

Enfatizamos tres ideas principales. Primero, en MRI es importante reducir el número de muestras adquiridas con el fin de reducir la duración de los exámenes clínicos. Dado que las máximas tasas de submuestreo (y por lo tanto las mayores reducciones de tiempo de adquisición) en CS se alcanzan con los métodos basados en la cuasi-norma $\mathrm{I}_{0}$, es relevante ahondar en su estudio. Segundo, si el número de muestras adquiridas es suficiente, los métodos de CS basados en la cuasi-norma $I_{0}$ producen reconstrucciones exactas y por lo tanto, sin artefactos en la imagen. Tercero, el número de muestras necesarias para reconstruir una imagen exacta depende de la imagen que se desea adquirir, la que es desconocida a priori. Por este motivo, en la práctica se desconoce cuántas muestras son necesarias para obtener una reconstrucción exacta. 
Nuestro trabajo extiende los resultados de Trzasko et al. al presentar una técnica de reconstrucción basado en una minimización por aproximaciones de la cuasi-norma I. Nuestro método, sin embargo, no requiere la definición de parámetros, y es por tanto más fácil de implementar. Adicionalmente, nuestro método permite evaluar un índice numérico, que no depende de información a priori de la imagen, que muestra una fuerte correlación con el error de reconstrucción, permitiendo determinar si la imagen submuestreada obtenida es idéntica a la original o no, y por tanto, si las tasas de submuestreo escogidas han sido excesivas o no.

\section{MATERIALES Y MÉTODOS}

Conceptos básicos de minimizaciones de la cuasi-norma $\mathrm{I}_{0}$ en Compressed Sensing.

Los métodos de reconstrucción de CS están diseñados para reconstruir imágenes que tengan muchos pixeles iguales a cero. Para explicar mejor este concepto podemos recurrir a un fantoma sintético de $10 \times 10$ pixeles para el cual graficamos sólo una línea (Figura 1). En la figura se puede apreciar que el valor de la cuasi-norma $I_{0}$ de la línea graficada es igual a cuatro, ya que sólo cuatro pixeles tienen una intensidad distinta de cero (en las posiciones 1 , $2,4$ y 6$)$.

Como es sabido, las imágenes de MRI no son adquiridas directamente en el espacio de la imagen, sino que en un espacio que corresponde a la Transformada de Fourier de ella. Por consiguiente, una vez que adquirimos la señal original de una MRI

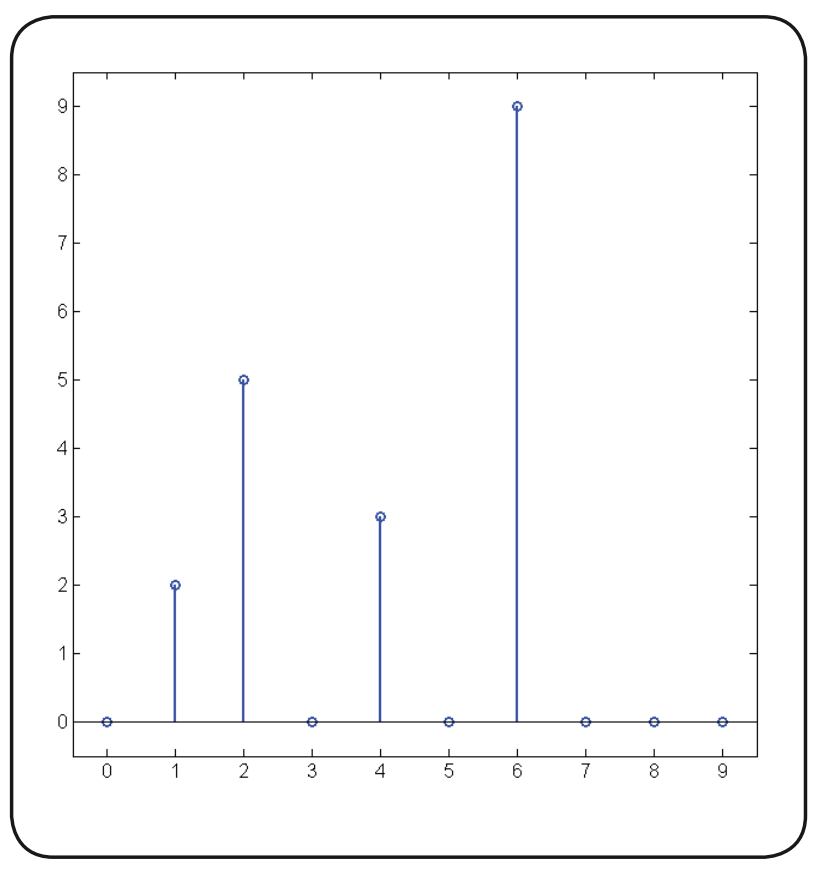

Figura 1. Línea de un fantoma de 10x10 pixeles. La cuasinorma $I_{0}$ de esta línea es cuatro, ya que sólo 4 de los 10 pixeles tiene una intensidad distinta de cero. debemos reconstruirla aplicando una transformada de Fourier inversa. En la forma estándar, debemos tomar tantas muestras en el espacio de Fourier, como pixeles queremos que tenga nuestra imagen. Una vez más podemos ejemplificar gráficamente estos conceptos con nuestro fantoma (Figura 2). Para poder ver nuestra línea de 10 pixeles, tenemos que obtener 10 muestras en el espacio de Fourier y luego aplicar una transformada de Fourier inversa (IFT).

El método de reconstrucción de CS mediante minimización de la cuasi-norma $I_{0}$ nos dice que basta con adquirir el doble de los pixeles distintos de cero para reconstruir la imagen deseada. En nuestro ejemplo del fantoma, esto corresponde a adquirir sólo ocho muestras (Figura 3), sin importar la ubicación de ellas. Esto equivale a una reducción del tiempo de adquisición de un $20 \%$. En el caso de la Figura 3 las ocho muestras escogidas se muestran en rojo y las no escogidas se muestran en azul.

El método original de reconstrucción de CS por minimización de la cuasi-norma $I_{0}$ consiste en probar todas las combinaciones de imágenes cuyas muestras en el espacio de Fourier coincidan con las muestras adquiridas (las muestras rojas en nuestro ejemplo de la Figura 3a) y elegir la que tenga un mayor número de pixeles iguales a cero en la imagen (Figura 3b). Formalmente esto equivale a evaluar cada pixel de la imagen (Figura $3 b$ ) en una función que valga 1 en todo lugar, salvo en el origen en cuyo caso la función vale cero (línea sólida negra en la Figura 4) y luego sumar los resultados originados con cada pixel. En la práctica, este procedimiento no es viable porque el número de combinaciones que debieran evaluarse es muy grande. Nuestro enfoque, al igual que el de Trzasko et al. ${ }^{(3)}$, es utilizar funciones continuas (líneas rojas en la Figura 4) con las que se pueda evaluar en forma aproximada la cuasi-norma I, y además resolver la minimización y reconstrucción de una manera factible y óptima. En la práctica esta minimización se resuelve de forma iterativa, en donde se resuelve una minimización local con una de las funciones continuas de aproximación y secuencialmente se va refinando la solución usando funciones más parecidas a la cuasi-norma $I_{0}$ (líneas rojas en la Figura 4).

Existen varias formas para realizar las minimizaciones locales, en nuestro caso utilizamos las mismas funciones de aproximación que Trzasko et al. ${ }^{(3)}$, pero proponemos una resolución de punto fijo, del tipo:

$$
C^{(n+1)}=-\left(P^{*} D^{(n)} P\right)^{-1} P^{*} D^{(n)} g^{(0)}
$$

donde $\mathrm{P}$ es una matriz que nos permite transformar las muestras no adquiridas $\mathrm{c}$ al espacio de Fourier; $\mathrm{P}^{*}$ es la matriz transpuesta conjugada de $\mathrm{P}$; $\mathrm{D}$ es una matriz diagonal que contiene información acerca de la pendiente de las funciones de aproximación; $\mathrm{g}^{(0)}$ es una imagen de inicialización arbitraria. La ecua- 

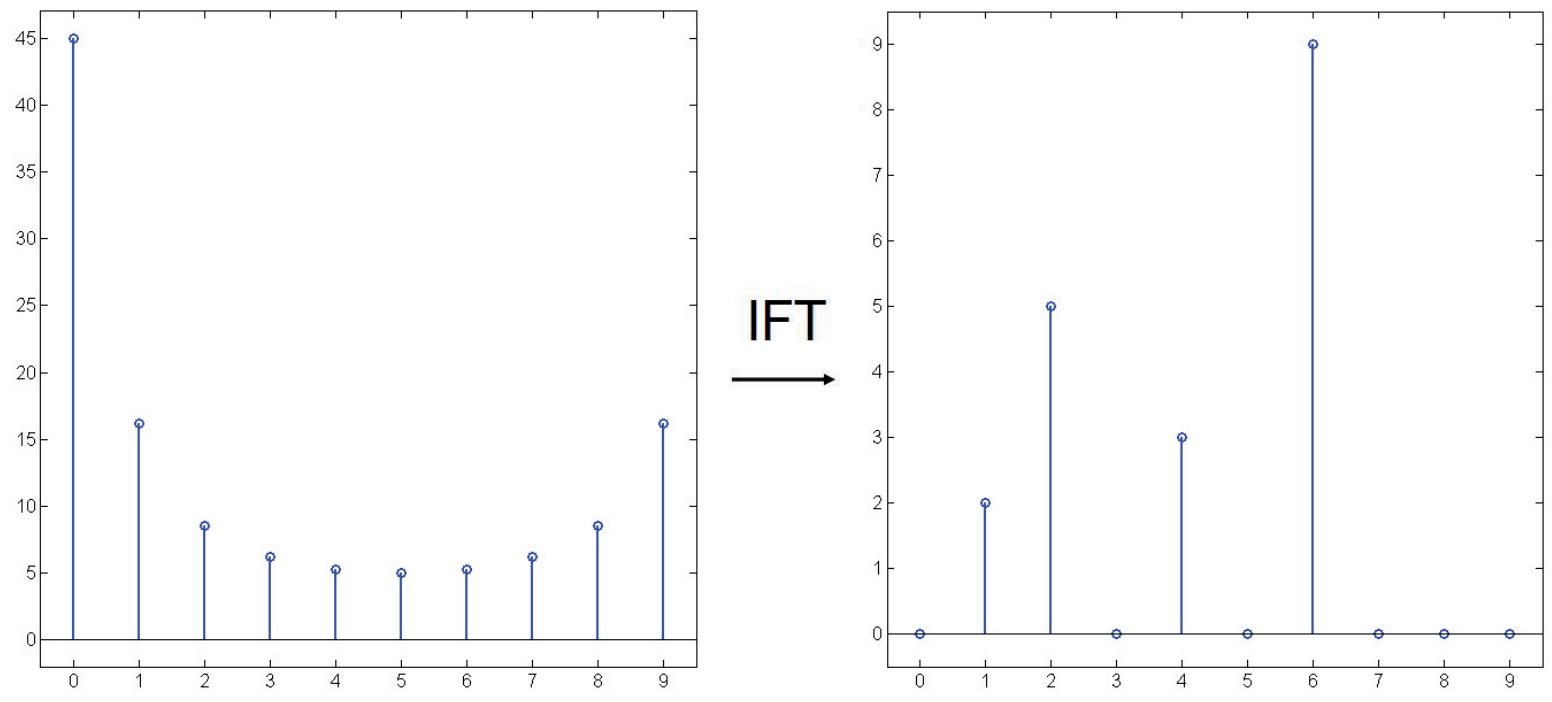

Figura 2. En RM las muestras se adquieren en el espacio de Fourier, por lo que es necesario reconstruirlas para poder ver una imagen; en aplicaciones estándares, este proceso se realiza aplicando una transformada de Fourier inversa a las muestras adquiridas. El número de muestras adquiridas debe ser igual al número de muestras que queremos que tenga la imagen reconstruida. Dado que nuestro fantoma tiene 10 pixeles, es necesario por lo tanto adquirir 10 muestras.

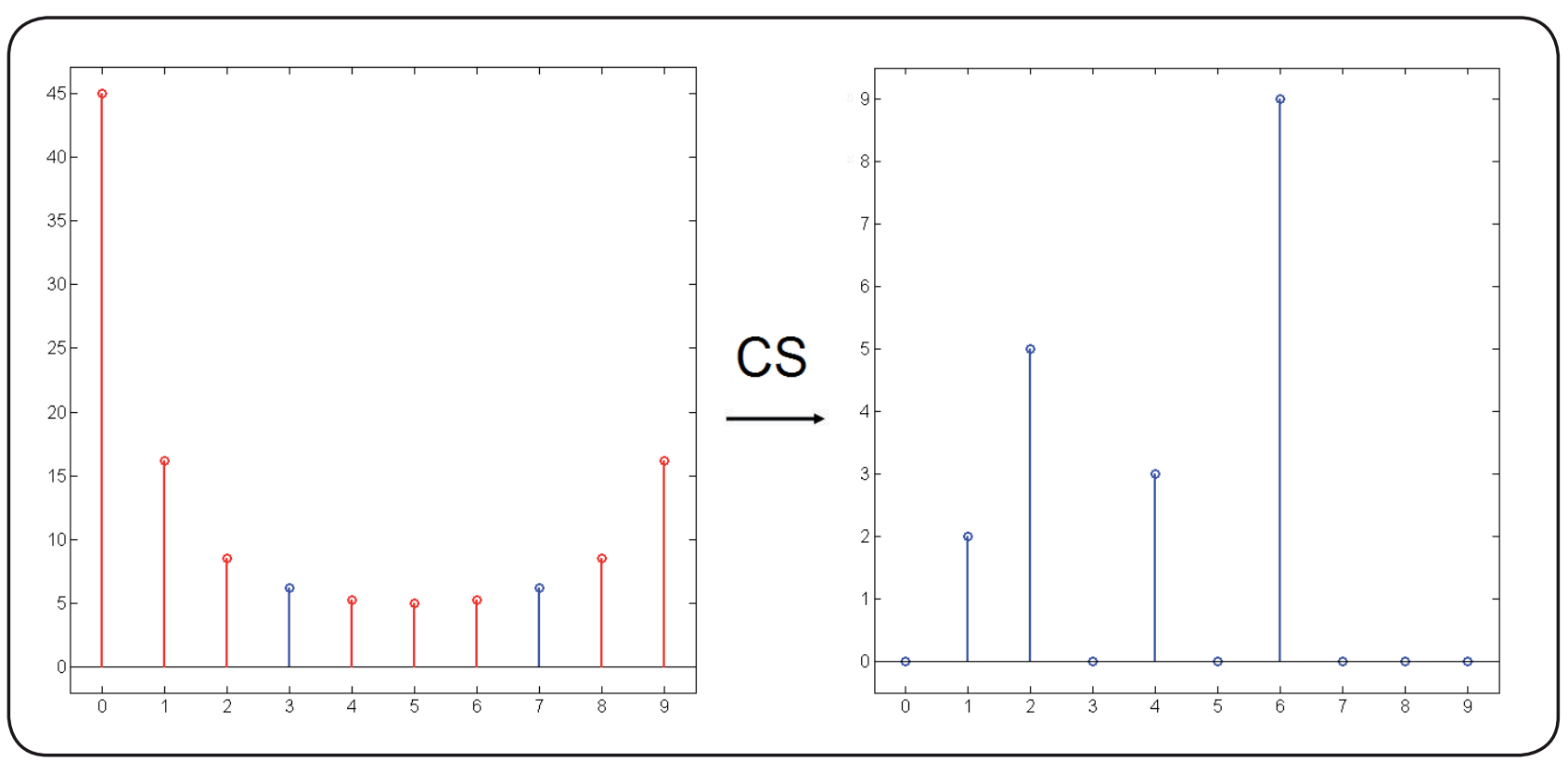

Figura 3. En reconstrucciones con Compressed Sensing, el número de muestras necesarias es el doble que el número de pixeles distintos de cero. Dado que nuestro fantoma tiene 4 pixeles distintos de cero, son necesarias sólo 8 muestras, por lo que logramos un $20 \%$ de reducción de tiempo de adquisición (las muestras pueden ser adquiridas en cualquier ubicación).

ción ${ }^{(1)}$ es un proceso iterativo que se inicializa con una imagen $\mathrm{g}^{(0)}$, que es reconstruida suponiendo que las muestras desconocidas son iguales a cero. Luego podemos calcular la cuasi norma $\mathrm{I}_{0}$ de $\mathrm{g}^{(0)}$ multiplicando por la matriz $\mathrm{D}$, y dado que los coeficientes desconocidos están en el espacio de Fourier, debemos multiplicar por la matriz de transformación P. Dado que sabemos que esta aproximación no es exacta, debemos compensar los errores inducidos por la aproximación multiplicando por la matriz $-\left(\mathrm{P}^{*} \mathrm{DP}\right)^{-1}$. Luego, debemos iterar hasta lograr que la diferencia de los valores encontrados de $\mathrm{c}$ en dos iteraciones consecutivas sea pequeña. Finalmente, con los valores encontrados de c y las muestras adquiridas, podemos reconstruir la imagen deseada aplicando la transformada de Fourier inversa. 


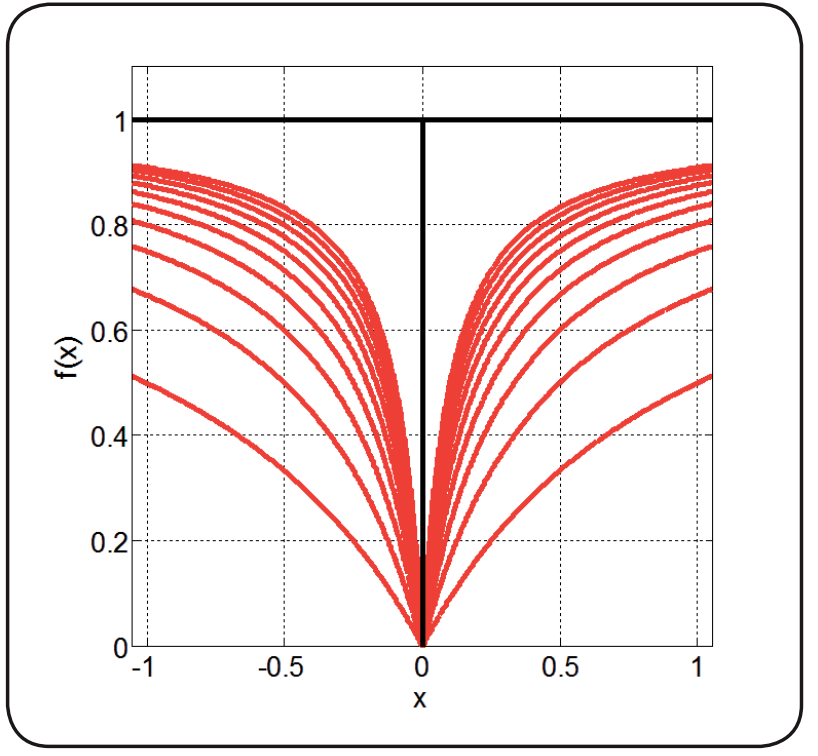

Figura 4. Compressed Sensing inicialmente fue formulado como un problema de minimización de la cuasi-norma $I_{0}$, graficada en línea negra. Debido a que este problema es de gran complejidad, se utilizan aproximaciones continuas cada vez más precisas, como por ejemplo aquellas graficadas en línea roja.

A diferencia del método de minimización propuesto por Trzsasko et al. ${ }^{(3)}$, en nuestro método no es necesario elegir ningún tipo de parámetro, los cuales por lo general dificultan las implementaciones ya que tienen que ser encontrados de manera heurística. Además, nos permite conocer un índice numérico de reconstrucción (RI), que se calcula como:

$$
R I=1-\frac{\lambda}{\mu}
$$

donde $\lambda$ y $\mu$ son el mínimo valor propio y el promedio de todos los valores propios de la matriz P*DP, respectivamente. Este índice RI tiene una alta correlación con el error de reconstrucción de las imágenes y no necesita ningún tipo de información acerca de la imagen que se quiere reconstruir, y por lo tanto, nos puede advertir sobre el uso de tasas de submuestreo excesivas.

\section{DESCRIPCIÓN DE LOS EXPERIMENTOS REALIZADOS}

Para verificar la exactitud de las reconstrucciones con nuestro método de CS, y para verificar si nuestro índice de reconstrucción (RI) se correlaciona con el error relativo de reconstrucción (RRE), realizamos dos experimentos.

En el primer experimento construimos fantomas de una dimensión, similares a los de la Figura 1, pero cuyo largo total $\mathrm{N}$ era de 97 pixeles. Variamos la cantidad de pixeles distintos de cero entre $1,5, \ldots, 89$ y 93 , y la cantidad de muestras adquiridas entre 2, 6, .., 90, 94. Para cada combinación se generaron 30 fantomas con valores aleatorios, los que se muestrearon en lugares aleatorios. Finalmente, se procedió a reconstruir los fantomas con nuestro método y para cada caso, se comparó nuestro índice de reconstrucción (RI) con el error relativo de reconstrucción (RRE).

En el segundo experimento se adquirió una imagen FLAIR de MRI con los siguientes parámetros: TR/TI/TE: 14679/3000/150 ms; flip angle 90; turbo

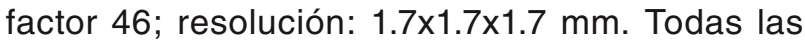
muestras necesarias de la imagen fueron adquiridas de tal forma de obtener una imagen de referencia para calcular el error de reconstrucción. Posteriormente, se descartaron en forma aleatoria $40 \%, 45 \%$ y $50 \%$ de las muestras para simular el proceso de submuestreo y por consiguiente, de reducción del tiempo de adquisición. Finalmente, se procedió a reconstruir las imágenes submuestreadas con nuestro método y para cada caso, se comparó nuestro índice de reconstrucción (RI) con el error relativo de reconstrucción (RRE). Para facilitar el análisis de los resultados cada línea de la imagen de MRI fue reconstruida en forma independiente.

Para ambos experimentos se evaluó la correlación entre el índice de reconstrucción RI y el error relativo de reconstrucción RRE utilizando curvas ROC (por sus siglas en inglés, Receiver Operating Characteristic).

Para efectos prácticos, el error relativo de reconstrucción RRE fue calculado como:

$$
R R E=\frac{\|g-f\|}{\|f\|}
$$

donde II II es el valor absoluto de la diferencia en niveles de gris de la imagen original $f$ y la reconstruida g, normalizada por los niveles de intensidad de la imagen $f$.

\section{RESULTADOS}

Considerando un $60 \%$ de las muestras la reconstrucción mediante CS es exacta para 164 de las 173 líneas que posee la imagen (Figura 5b), es decir no existe diferencia alguna entre las líneas reconstruidas de la manera tradicional con todas las muestras, y las líneas reconstruidas con CS y sólo un $60 \%$ de las muestras. Esto se puede apreciar con mayor detalle cuando graficamos una línea específica de la imagen (Figura 6a). Las 9 líneas que presentan diferencias se debe a que el submuestreo fue excesivo, por lo que sólo bastaría con tomar un poco más del $60 \%$ de las muestras para obtener una imagen idéntica a la imagen estándar. 
Considerando ahora sólo un $55 \%$ de las muestras, existe un mayor número de líneas en donde el submuestreo es excesivo, por lo que aparecen un número mayor de líneas con distorsiones (Figura 5c). Esto se puede apreciar mejor cuando graficamos una línea de la imagen (Figura 6b).

Finalmente, cuando consideramos sólo el $50 \%$ de las muestras aún se conserva gran parte de la estructura de la cabeza, pero el número de líneas con problemas se
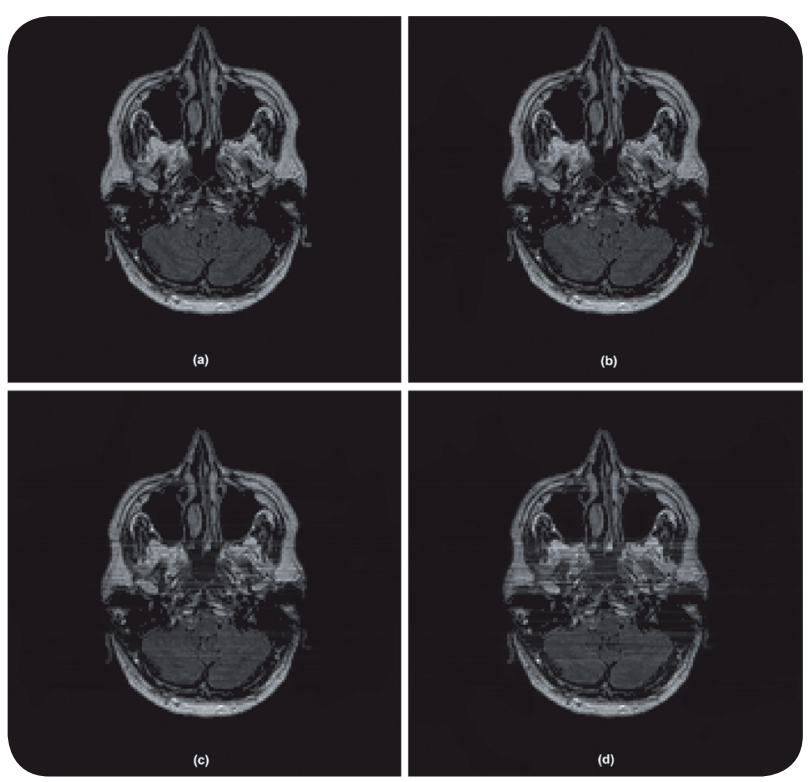

Figura 5. Reconstrucción de una imagen de RM utilizando Compressed Sensing. (a) imagen original; (b) reconstruida en forma exacta con sólo el $60 \%$ de las muestras, salvo para 9 líneas en donde existen algunas diferencias producto de que el muestreo fue excesivo para dichas líneas; (c) imagen reconstruida con el $55 \%$ de las muestras, es posible apreciar líneas en que los artefactos de reconstrucción son visibles; (d) imagen reconstruida con el $50 \%$ de las muestras, los artefactos de reconstrucción son claramente visibles. hace ostensiblemente mayor (Figura 5d), y la magnitud de las diferencias también se incrementa (Figura 6c).

El índice de reconstrucción $\mathrm{RI}$ entrega valores normalizados entre 0 y 1 , y como se aprecia en la Figura 7, los valores de RI lejanos a 1 indican que los errores cometidos al reconstruir (RRE) son bajos 0 nulos. Por consiguiente, valores de RI lejanos de 1 nos indican que las tasas de submuestreos han sido adecuadas. Por el contrario, cuando RI se acerca a 1 , nos indica que existen errores de reconstrucción, y por lo tanto que las tasa de submuestreo han sido excesivas. Esta observación es válida tanto para el fantoma como para la imagen de MRI.

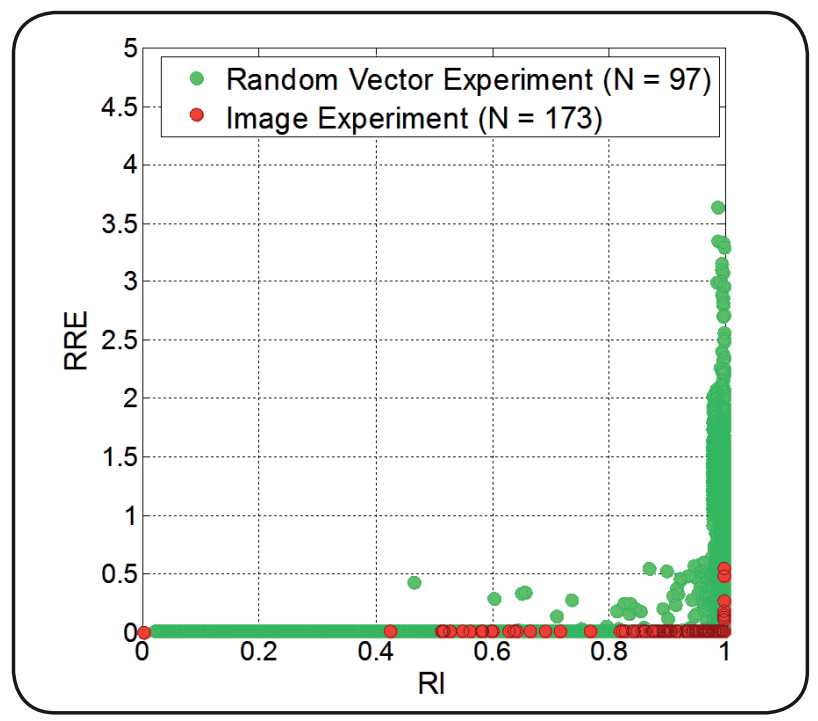

Figura 7. Para valores del índice de reconstrucción RI lejanos a 1, los errores cometidos al reconstruir (medidos como RRE) son bajos o nulos, indicando que las tasas de submuestreos han sido adecuadas. Cuando RI se acerca a 1, indica que existen errores de reconstrucción, y por lo tanto que las tasas de submuestreo han sido excesivas. Esta observación es válida tanto para el fantoma como para la imagen de RM.

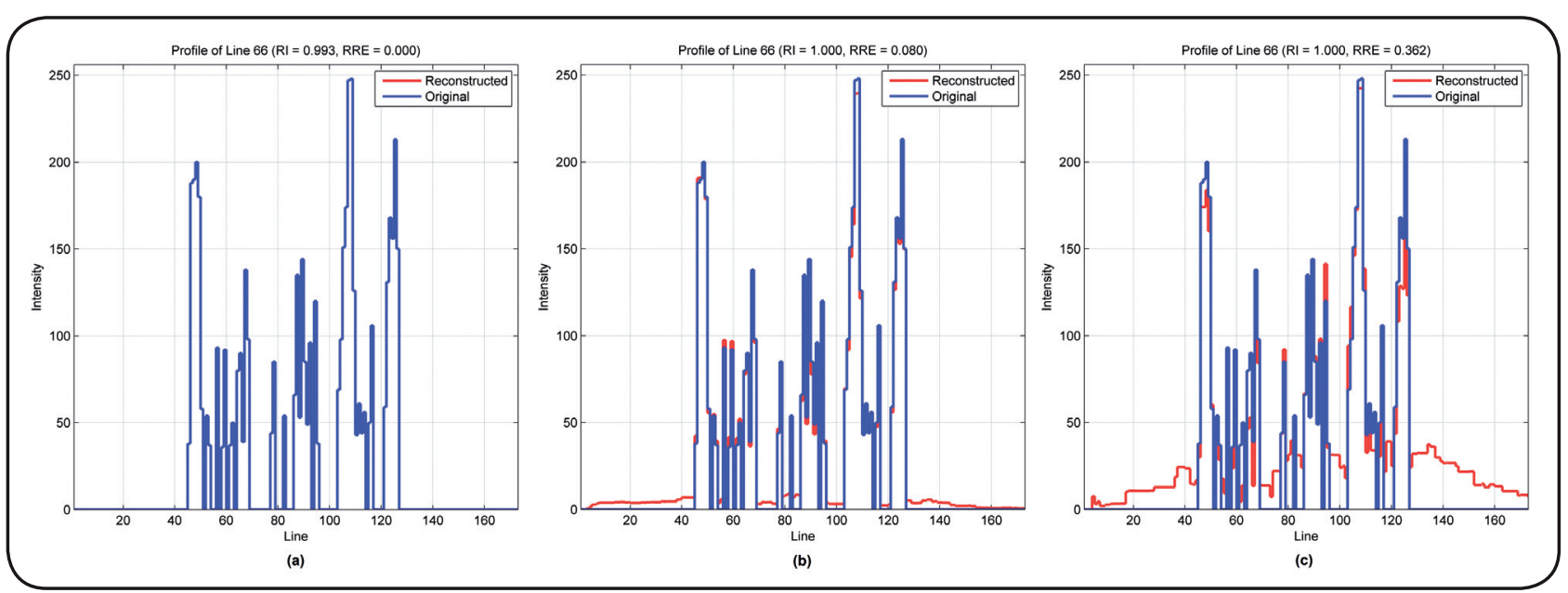

Figura 6. Perfiles de intensidad de una línea de la imagen original y reconstruida. Es posible apreciar en el panel (a) que los perfiles de la imagen original y la reconstrucción con el $60 \%$ de las muestras son exactas. En el panel (b) se puede apreciar que con el $55 \%$ de los coeficientes las diferencias comienzan a ser visibles. Finalmente, en el panel (c) se puede apreciar que existen artefactos visibles cuando no se han adquirido suficientes muestras. 
El valor predictor del índice $\mathrm{RI}$ es alto y tiene un punto óptimo en torno a $\mathrm{RI}=0,95$ para el cual la tasa de falsos positivos es inferior al $5 \%$ y de positivos verdaderos es superior al 95\% (Figura 8 ). Esto quiere decir que con aproximadamente un $95 \%$ de probabilidad podemos saber si una línea de la imagen fue reconstruida con errores o no, y por lo tanto si necesitamos aumentar el número de muestras adquiridas.

\section{CONCLUSIÓN}

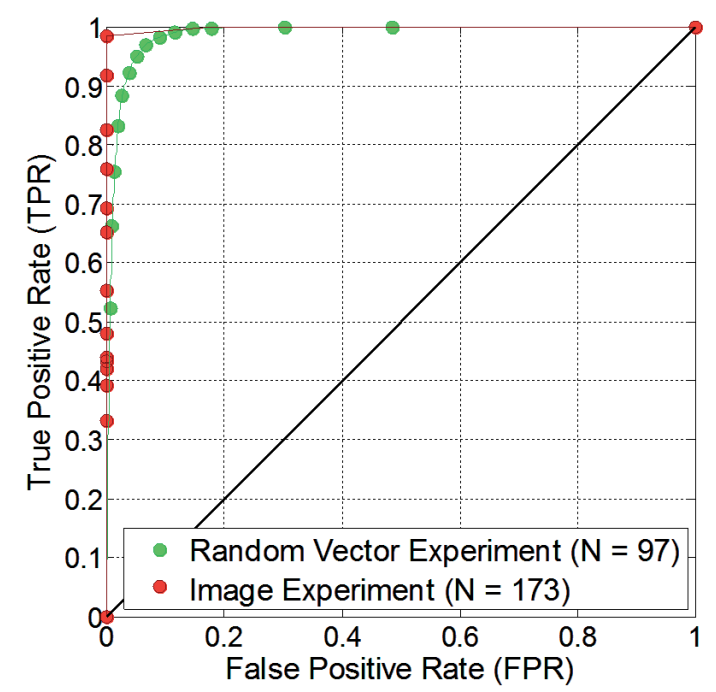

Figura 8. El índice RI tiene un alto valor predictor sobre el error de reconstrucción RRE. Si escogemos el punto óptimo de la curva ROC $(R I=0,95)$, con un $95 \%$ de probabilidad aproximadamente, podemos saber si una línea de la imagen fue reconstruida con errores o no, y por lo tanto si necesitamos aumentar el número de muestras adquiridas.

Nuestro trabajo propone un método de reconstrucción que constituye una mejora en relación a los métodos presentes en la literatura. Este método consiste en un problema de minimización que: no tiene parámetros libres, satisface exactamente la consistencia de datos y la complejidad del método es proporcional a la tasa de submuestreo. Adicionalmente, nuestros resultados muestran que el método es equivalente en la práctica a resolver la minimización basada en la cuasi-norma I.

A partir de este método, definimos un índice de reconstrucción que exhibe una fuerte correlación con el error de reconstrucción. Este índice no requiere ninguna información a priori de la imagen a adquirir $y$, por tanto, puede ser utilizado para determinar si la imagen submuestreada es igual a la original. El método de detección propuesto con este fin, permite obtener una alta tasa de detecciones correctas con valores pequeños de falsas detecciones. Consecuentemente, esto permite determinar si la tasa de submuestreo empleada es excesiva y debe ser ajustada.

\section{AGRADECIMIENTOS}

Este trabajo cuenta con el financiamiento de FONDECYT (\#1070674).

\section{BIBLIOGRAFÍA}

1. Candès, E., Romberg, J., Tao, T. Robust uncertainty principles: exact signal reconstruction from highly incomplete frequency information. IEEE Trans. Inf. Theory 2006; 52: 489-509.

2. Lustig, M., Donoho, D., Pauly, J.M. Sparse MRI: the application of Compressed Sensing for rapid MR imaging. Magnetic Resonance in Medicine 2007; 58: 1182-1195

3. Trzasko, J., Manduca, A. Highly undersampling magnetic resonance image reconstruction via homotopic I - -minimization. IEEE Trans. Med. Imag. 2009; 28: 106-121.

4. Mohimani, G.H., Babaie-Zadeh, M., Jutten, C. A fast approach for overcomplete sparse decomposition based on smoothed I -norm. IEEE Trans. Signal Process.2009; 57: 289-301. 